Husar, Peter; Berkes, Sebastian; Götze, Andreas; Henning, Günter; Plagwitz, KaiUwe:

\title{
Verbesserung des SNR bei mehrkanaligen EEG-Ableitungen
}

\author{
Zuerst erschienen in: Biomedizinische Technik = Biomedical Engineering. - Berlin [u.a.] : \\ de Gruyter. - 47 (2002), S1b, S. 566-569. \\ Jahrestagung der Deutschen Gesellschaft für Biomedizinische \\ Technik (DGBM) im VDE ; 36 (Karlsruhe) : 2002.09.25-27 \\ Erstveröffentlichung: 2002 \\ Datum Digitalisierung: $\quad 2009-11-26$ \\ ISSN (online): $\quad$ 1862-278X \\ ISSN(print) 0013-5585 \\ DOI: $\quad$ 10.1515/bmte.2002.47.s1b.566 \\ [Zuletzt gesehen: 2019-12-06]
}

„Im Rahmen der hochschulweiten Open-Access-Strategie für die Zweitveröffentlichung identifiziert durch die Universitätsbibliothek IImenau."

"Within the academic Open Access Strategy identified for deposition by IImenau University Library."

„Dieser Beitrag ist mit Zustimmung des Rechteinhabers aufgrund einer (DFGgeförderten) Allianz- bzw. Nationallizenz frei zugänglich."

„This publication is with permission of the rights owner freely accessible due to an Alliance licence and a national licence (funded by the DFG, German

Research Foundation) respectively."

\section{DFG}

Nationallizenzen 


\title{
VERBESSERUNG DES SNR BEI MEHRKANALIGEN EEG-ABLEITUNGEN
}

\author{
P.Husar, S.Berkes, A.Götze, G.Henning, K.U.Plagwitz \\ Institut für Biomedizinische Technik und Informatik, Technische Universität Ilmenau, Deutschland
}

peter.husar@tu-ilmenau.de

\begin{abstract}
The main problem in measurements of the focal VEP (Visual Evoked Potential) is its weak SNR (Signal-to-Noise Ratio). The most common method for enhancement of the SNR is the stimulus synchronized averaging. For study of single responses other ways in SNR improvement are needed. In this contribution a new method based on space-time selective measurement is introduced, which can be interpreted as beaming a signal source. Since the anatomical structures of sources generating the focal VEP are known in general and if the electrode positions are of sufficient density over the visual cortex, a source beamer can be realized by controlling the channels' delays.

Keywords - visual evoked potential, beam forming, sensor array, multichannel processing
\end{abstract}

\section{Einleitung}

Visuell evozierte Potentiale (VEP) sind ein wichtiger objektiver Indikator in der Funktionsdiagnostik des visuellen Systems. Sie können und sollen nicht die bewährten Methoden der neurologischen und/oder der ophthalmologischen Funktionsprüfung ersetzen. Vielmehr sollen sie eine Möglichkeit zur Objektivierung der Untersuchung in Fällen bieten, in denen die Kommunikation mit dem Untersuchten gestört ist, d.h. in Fällen, in denen sie nicht möglich oder nicht gewollt ist.

Aus der Sicht der Grundlagenforschung sind die VEP vom enormen Interesse, da sie Einblicke in die Funktionen des visuellen Systems ermöglichen, die subjektiv nicht quantifizierbar oder gar nicht erst wahmehmbar sind.

Die verbreitetste Methode ist - nicht nur weil sie sehr einfach realisierbar ist - die reizsynchrone Mittelung von Einzelantworten. Diese in der VEP-Forschung seit über vierzig Jahren angewandte Methode zählt auch im technischen Bereich bezüglich der Anhebung des SNR zu den effektivsten. Allerdings sind die auf dieser Methode beruhenden Ergebnisse nur Aussagen über das mittlere zeitliche Verhalten. Betrifft die diagnostische Fragestellung die zeitliche Entwicklung eines VEP-Parameters, z.B. die Veränderung der Latenz einer Welle, so ist diese Frage mit der reizsynchronen Mittelung nicht zu beantworten. Hinzu kommt, dass bei Untersuchungen, bei denen ein Parameter über einen größeren Bereich variiert werden muss, z.B. in der Perimetrie die Position des kleinflächigen Lichtflecks, diese Methode zur kumulativen Verlängenung der Untersuchungsdauer fuihrt, was aus der medizinischen Sicht selten akzeptiert wird.

Daher ist es notwendig - ob im Interesse der Verkürzung der Untersuchungsdauer oder um zeitliche Verläufe der interessierenden Parameter wu chalten - Methoden der
SNR-Verbesserung einzusetzen, die die notwendige Messzeit deutlich verkürzen.

Bei mehrkanaligen Ableitungen bietet sich prinzipiell die Möglichkeit, das Ensemble-Mittel zu berechnen. Dies führt theoretisch zur SNR-Anhebung in Echtzeit, für die sonst eine der Kanalzahl entsprechende Anzahl von Einzelantworten notwendig wäre. Allerdings sind die signaltheoretischen Voraussetzungen für die SNR-Anhebung mit Hilfe des Ensemblemittels bei realen Signalen nur unzureichend erfilltt. Mit Methoden der Signalverarbeitung im Verbundbereich Raum-Zeit können die Signaleigenschaften verbessert werden.

\section{Methoden}

Gehen wir von einem additiven Modell (Gl. 1) für das gesuchte zeitdiskrete transiente Signal $s(k)$ und weißes Rauschen $n(k)$ aus, so wird nach der Mittelung von $N$ sukzessiven Realisierungen ein SNR entsprechend Gl. 2 erreicht.

$$
\begin{gathered}
x(k)=s(k)+n(k) \\
S N R=N \cdot S N R_{0}, \quad S N R_{0}=\sum_{k=1}^{M} s^{2}(k) / \sum_{k=1}^{M} n^{2}(k)
\end{gathered}
$$

Das in Gl. 1 formulierte Modell kann auch als eine Momentaufnahme eines mehrkanaligen Signals interpretiert werden. Wird hier ein mitlaufendes Ensemblemittel gebildet, so wird entsprechend Gl. 2 eine SNR-Verbesserung in Echtzeit erzielt.

In der Realität muss man damit rechnen, dass das Signal in den Kanälen nicht synchron und mit unterschiedlichen Amplituden auftritt. Dementsprechend wird das Signalmodell aus der Gl. 1 angepasst (Gl. 3); $i$ ist der Kanalindex, $\tau$ die Zeitverschiebung.

$$
\begin{aligned}
& x_{i}(k)=A_{i} s_{i}\left(k+\tau_{i}\right)+n_{i}(k) \\
& \bar{x}(k)=\frac{1}{N} \sum_{i=1}^{N} x_{i}(k)
\end{aligned}
$$

Die Kanalamplituden $A_{i}$ sind Gegenstand der Optimienıng eines räumlichen Filters und werden in diesem Beitrag nicht berücksichtigt. Die Kanalverzögenungen $\tau_{i}$ fiihren rur Herabsetzung des theoretisch erreichbaren SNR ((il. 2), im Extremfall sogar nur Abschwächung. Dics folgt aus der (il. 4 für das Ensemblemittel, wobei $s(k)$ stets als transientes Signal anzunehmen ist.

Die Kanalverzögenungen ergeben sich aus der Projectiou der räumlichen von der Signalquelle ausgegchenden Wielle 
auf dic Sensorenflächc. Im allgemeinen hängen die Eigenschaften der Welle von der Ausbreitungsgeschwindigkeit im Medium und von der Bewegung der Quelle ab. Sind die Aushreilungsgescliwindigkeit und dic Sensorpositionen bekannt, können dic Kanalverzögerungen gezielt gesteuert werden, um cine gewünschte Richtcharakteristik des Sensorfeldes zu erreichen. Dies ist cin übliches Verfahren bei Radar, Sonar und Richtantennen.

Bei der EEG-Analyse gestaltet sich dieses Problem wesentlich schwieriger: Dic Ausbreitungsgeschwindigkeit der elektrischen Felder ist so hoch, dass die Kanalverzögerungen allein durch die Quellenbewegung bestimmt werden. Dic Qucllengeschwindigkeit - also die Ausbreitungsgeschwindigkeit der Reize auf neuronalen Bahnen - variiert über einen großen Bereich von $0.1 \mathrm{~m} / \mathrm{s}$ bis $10 \mathrm{~m} / \mathrm{s}$, wobei die Angaben in der Literatur stark differieren, z.B. [1], [2]. Daher muss sie als unbekannt Digefionsonpositioden lassen sich relativ genau nach anatomischen Merkmalen (z.b. Nasion, Inion beim 10-20System) festlegen. Diese Positionen variieren interindividuell stark im Vergleich zu kortikalen Strukturen, so dass eine gezielte Fokussierung ohne Zusatzmaßnahmen (MRI mit EEG) kaum möglich ist. Aus diesen Gründen ist es notwendig, Verfahren zu entwickeln, die sich an die aktuelle anatomische und elektrophysiologische Situation anpassen.

Herkömmliche Verfahren der adaptiven Beamformung kommen aus den o.g. Gründen nicht in Frage, für klassische auf der Minimierung einer Fehlerfunktion basierende adaptive Filterung fehlt die Signal- bzw. die Rauschreferenz [3]. Ein neuer praktikabler Ansatz beruht auf der folgenden Überlegung: Das primäre Ziel ist die Maximierung des SNR (Gl. 5).

$$
S N R \rightarrow \max , \frac{\partial S N R}{\partial \tau_{i}}=0, \frac{\partial^{2} S N R}{\partial \tau_{i}^{2}}<0
$$

Nehmen wir zunächst an, dass das transiente Signal $s(k)$ ein einphasiges Signal ist, d.h. es besitzt nur einen Extremwert. Als Beispiel soll hier ein verrauschter Gaußscher Impuls dienen:

$$
x_{i}(k)=A_{i} \exp \left(-b\left(k+\tau_{i}\right)^{2}\right)+n_{i}(k)
$$

Wegen des stochastischen Anteils muss auf Gl. 6 der Erwartungsoperator angewendet werden, so dass Gl. 5 unter Verwendung von Gl. 6 sich zur Gl. 7 modifizieren lässt:

$$
E\left\{\bar{x}^{2}(k)\right\} \rightarrow \max , \frac{\partial E\left\{\bar{x}^{2}(k)\right\}}{\partial \tau_{i}}=0, \frac{\partial^{2} E\left\{\bar{x}^{2}(k)\right\}}{\partial \tau_{i}^{2}}<0
$$

Aus Gl. 7 ergibt sich für $d_{i}$ die analytische Lösung für alle einphasigen Signale $\left(d_{i}\right.$ ist die zu realisierende Verzögerung im Kanal $i$ ):

$$
d_{i}=-\tau_{i} \quad b z w . \quad \vec{d}=-\vec{\tau}
$$

Die Lösung nach Gl. 8 ist praktisch nur umsetzbar, wenn die Signalform bekannt ist. Dies ist jedoch bei realen EEGSignalen nicht der Fall. Daher kann das Maximum iterativ gesucht werden:

$$
\vec{d}_{j+1}=\vec{d}_{j}+\mu \nabla_{j},
$$

wobci $\mu$ dic Adaptionskonstante ist und $\nabla$ den Gradienten des SNR bezüglich der Kanalverzögerung repräsentiert:

$$
\nabla_{j}=\left\{\begin{array}{c}
\partial S N R / \partial \tau_{1} \\
\vdots \\
\partial S N R / \partial \tau_{N}
\end{array}\right\}, \quad \hat{\nabla}_{j}=\left\{\begin{array}{c}
\Delta S N R / \Delta \tau_{1} \\
\vdots \\
\Delta S N R / \Delta \tau_{N}
\end{array}\right\}
$$

Der Gradient kann für deterministische Signale berechnet werden, für unbekannte Signalverläufe wird er geschätzt (Gl. 10). Um Instabilitäten zu vermeiden, wird der Gradient auf seine maximale Komponente normiert

$$
\hat{\nabla}_{j}^{*}=\hat{\nabla}_{j} / \max \left(\left|\hat{\nabla}_{j}\right|\right),
$$

dann kann $\mu$ unabhängig vom aktuellen Signal im Bereich $\mu \in\langle 0 ; 1\rangle$ gewählt werden.

\section{Ergebnisse}

\section{Simulierte Signale}

Entsprechend dem Signalmodell in Gl. 6 wurde ein 16kanaliges Signal simuliert, siehe Abb. 1.

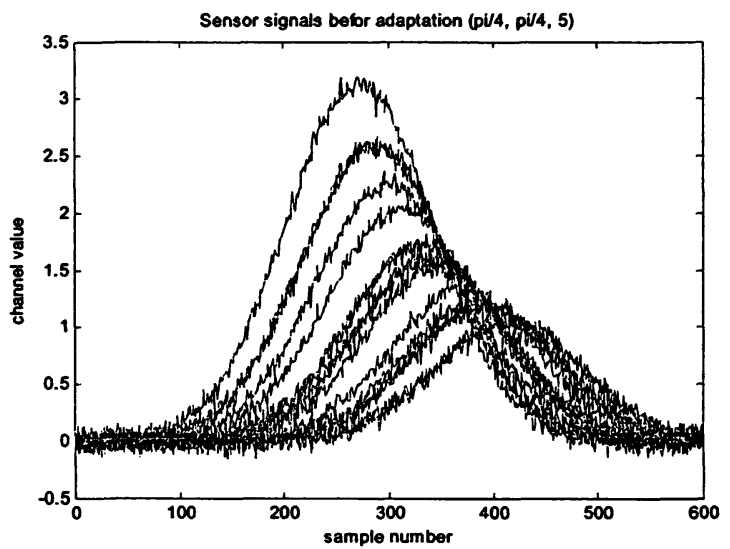

Abb. 1: 16-kanaliger verrauschter Gaußimpuls. Oktogonale Sensormatrix mit einem Abstand zwischen den Sensoren von $3 \mathrm{~cm}$ (Abb. 4). Signalquelle mit Kugelkoordinaten (phi, theta, $r)=(\pi / 4, \pi / 4,5 \mathrm{~cm})$ bezïglich des Sensornullpunktes. Ausbreitungsgeschwindigkeit $\mathrm{c}=1 \mathrm{~m} / \mathrm{s}$.

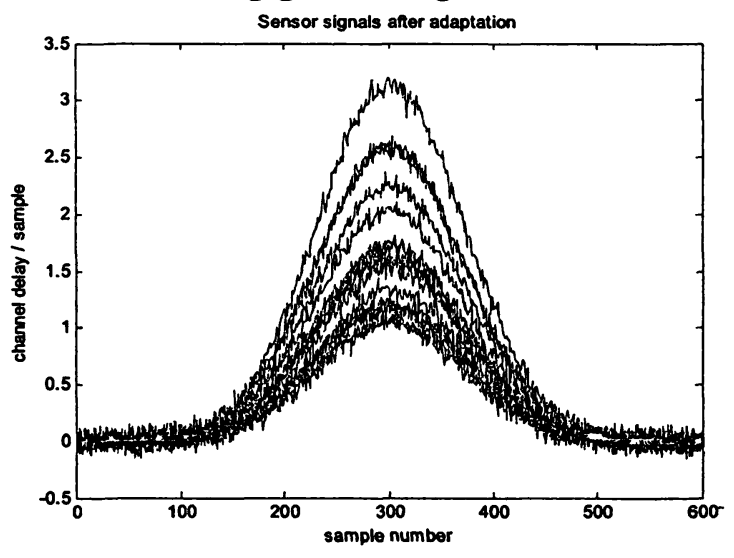

Abb. 2: Signal in Abb. 1 nach abgeschlossener Adaptation 
Unter Anwendung der Gln. 9,10,11 wurde der adaptive Algorithmus zur Bestimmung der zusätzlichen Kanalverzögerungen $d_{i}$ angewendet, das Resultat nach der abgeschlossenen Adaptation ist in der Abb. 2 dargestellt. Die Adaptation erfolgt unter diesen idealen Bedingungen relativ schnell. In dem simulierten Beispiel wird das SNRMaximum nach etwa 70 Iterationsschritten erreicht, siehe Abb. 3.

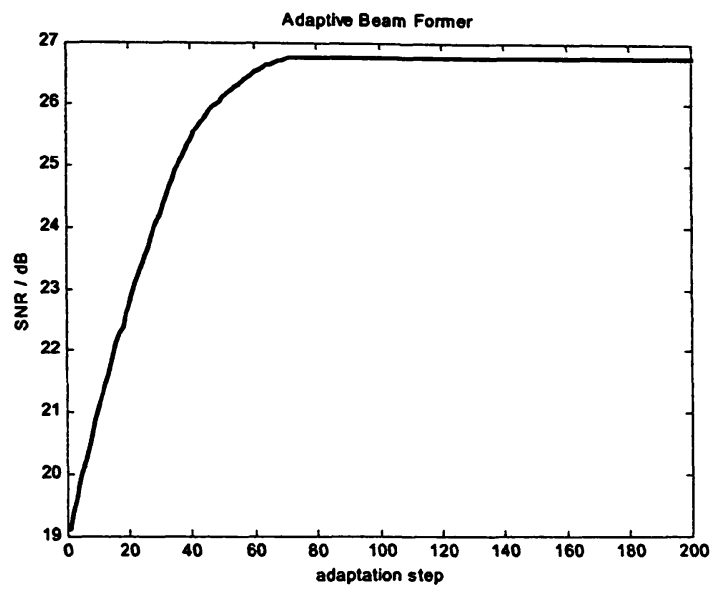

Abb. 3: SNR-Verlauf während der Adaptation

Die geschätzten Kanalverzögerungen nach abgeschlossener Adaptation gibt die Abb. 4 an. Der mittlere relative Schätzfehler der Kanalverzögerung betrug 1,6\% mit einer Standardabweichung von $3,8 \%$.

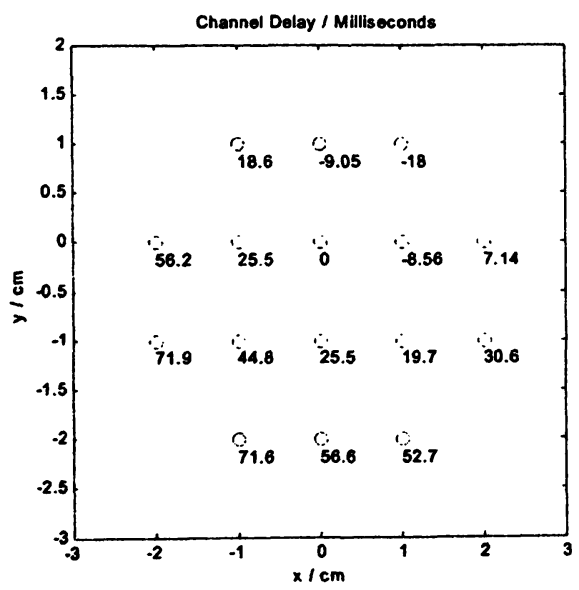

Abb. 4: Kanalverzögerungen nach abgeschlossener Adaptation der Signalquelle in Abb. 1.

Wie Abb. 4 zeigt, kann die Kanalverzögenung negativ werden. Das bedeutet, dass das Signal an diesen Sensoren früher ankommt (Kanäle 2,3,7), als im Nullpunkt des Sensorfeldes (Kanal 6). Für den praktischen Einsatz bedeutet das aus der Sicht der Kausalität jedoch kein Problem. Man führt durch eine einfache Translation des Verzögerungsvektors auf der Zeitachse eine zusätzliche Verzögerung so ein, dass alle Kanalverzögenungen positiv werden.

\section{Reale Sigmale}

Die in Abb. 4 dargestellte Sensormatrix wurde über dem visuellen Kortex so angeordnch, dass der Elcktrodenab- stand $10 \%$ des Abstandes Nasion-Inion betrug. Kanal 15 (untere Reihe, Mitte) lag über dem Inion, Kanal 11 (zweite Reihe von unten, Mitte) über $\mathrm{Oz}$, Kanal 2 (erste Reihe von oben, Mitte) über $\mathrm{Pz}$ des 10-20-Systems. Zur optischen Stimulation wurde ein herkömmliches Perimeter mit lichtstarken Leuchtdioden verwendet. Die Leuchtdichte betrug $2000 \mathrm{~cd} / \mathrm{m}^{2}$, die Impulsdauer war $20 \mathrm{~ms}$. Für die reizsynchrone Mittelung wurden 64 Einzelantworten aufgenommen. Die Aufnahmen enthalten pre- und poststimulative EEG-Abschnitte von jeweils einer halben Sekunde Länge. Das SNR wurde entsprechend dem Signalmodell (Gl. 1) nach Modifikation der Gl. 2 geschätzt (Gl. 12):

$$
S \hat{N N R}=\frac{\sum_{k=-p r e}^{0} x^{2}(k)}{\sum_{k=1}^{\text {post }} n^{2}(k)}-1, \quad x(k)=E E G_{\text {post }}+V E P
$$

Der Adaptationsalgorithmus wurde auf reale Daten (zentraler Reiz, Sichtwinkel $0.2^{\circ}$ ) angewendet. Zunächst wurde die CAR (Common Average Reference) abgezogen, anschließend wurden die Daten über 64 Einzelantworten gemittelt. Dies war notwendig, um für die Adaptation ein hinreichendes SNR zu erreichen. Das 16-kanalige VEP vor und nach der Adaptation ist in Abb. 5 dargestellt.
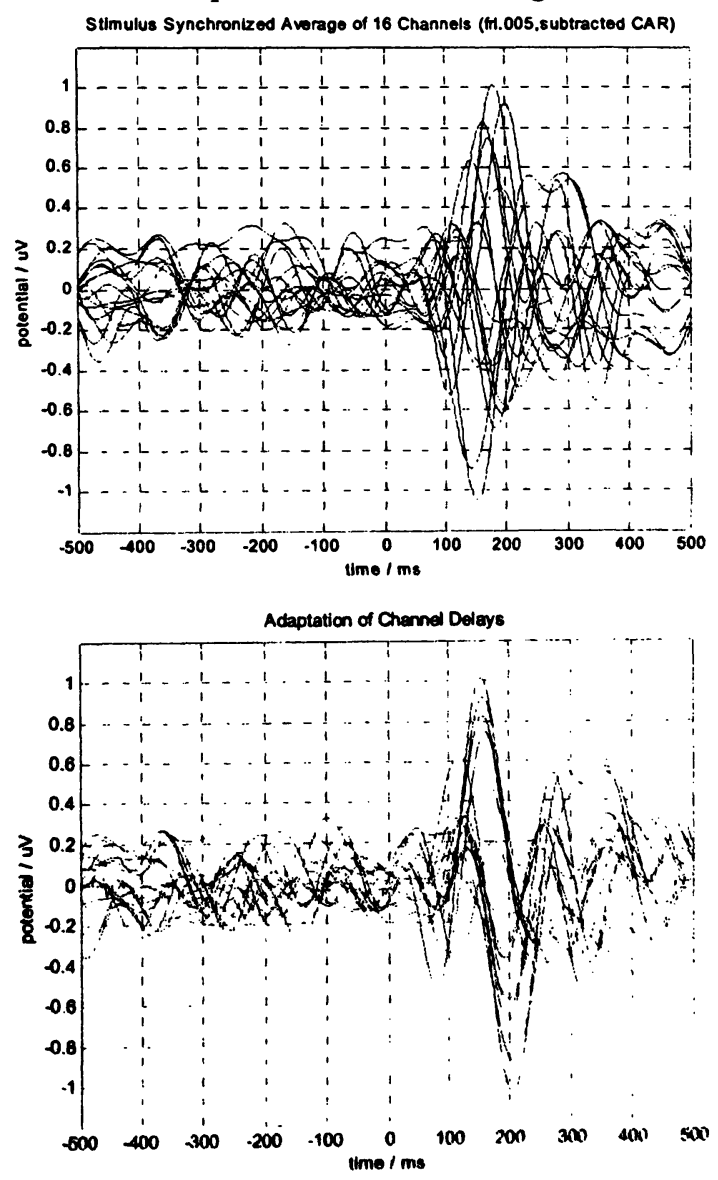

Abb. 5: 16-kanaliges über 64 Einzelantworten ncizs!nchron gemitteltes VEP mit abgerogener (.AR vir der Adaptation (ohen) und nach abschlossencr Adaptation (unten). 


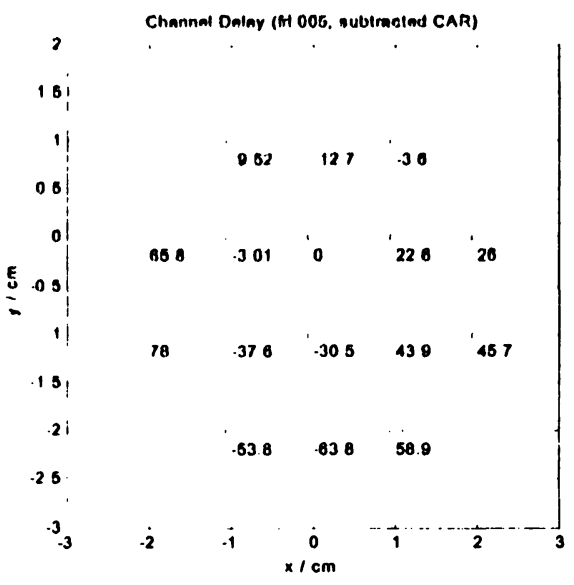

Abb. 6: Kanalverzögerungen nach abgeschlossener Adaptation in Millisekunden.

Die Adaptation wurde nach 100 Schritten abgebrochen, die ermittelten Kanalverzögerungen gibt Abb. 6 an.

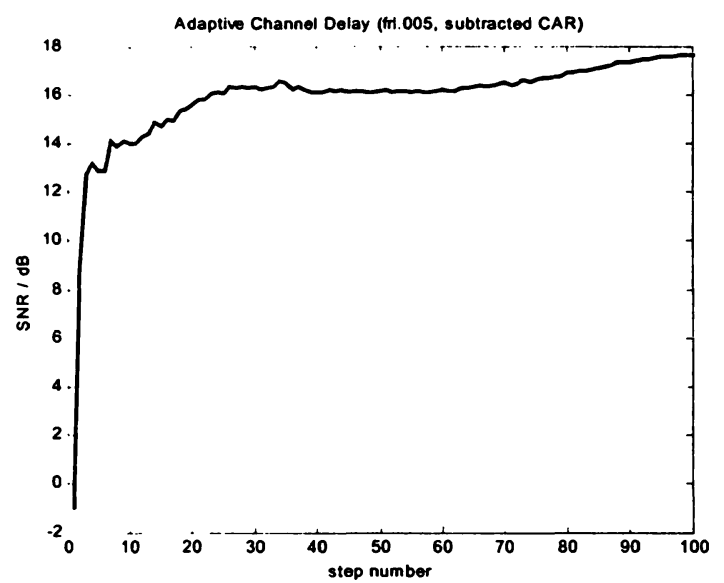

Abb. 7: SNR-Verlauf während der Adaptation realer Daten

Die SNR-Verbesserung erreicht nicht die Werte der simulierten Signale (Abb. 7), ihr Verlauf in Abhängigkeit von der Anzahl der Adaptationsschritte ist nicht monoton steigend.

\section{Diskussion}

Das theoretisch erreichbare Maximum der SNRVerbesserung von $12 \mathrm{~dB}$, das sich aus der Ensemblemittelung über 16 Kanäle (simultanes Mittel) ergibt, wird selbst bei simulierten Daten nicht erreicht. Das liegt daran, dass zwar das Rauschen nahezu ideale Eigenschaften besitzt (normalverteilt, weiß), aber die Signalparameter in den Kanälen relativ stark variieren. In diesem konkreten Beispiel erreicht die SNR-Verbesserung nach der Adaptation einen Wert von ca. 8dB. Eine weitere Anhebung wäre mit Hilfe eines räumlichen Filters denkbar. Dies ist Gegenstand laufender Untersuchungen.

Bei realen Daten sind die Signaleigenschaften des gesuchten VEP sowie des spontanen EEG erwartungsgemäß viel schlechter als bei simulierten Daten. Bei einer unipolaren EEG-Ableitung (Sensoren in Abb. 6 gegen verbundene Ohren) ist die räumliche Korrelation nicht nur beim VEP stark, sondern auf beim spontanen EEG. Daher führt die Adaptation zwar zur Deckung der VEP-Wellen ähnlich wie in Abb. 2, aber kaum zur Unterdrückung des Rauschens. Die SNR-Verbesserung ist in diesem Fall unwesentlich. Eine signifikante Reduktion der räumlichen Korrelation wird crst durch den Abzug der CAR (Common Average Referenz) erreicht. Allerdings fuihrt dies dazu, dass die VEP-Wcllen zum Teil unterschiedliches Vorzeichen besitzen bzw. im spontanen EEG auf Grund unzureichender Amplitude verschwinden (Abb. 5).

Der steile Anstieg des SNR zu Beginn der Adaptation (Abb. 7) kann nicht dem Algorithmus zugeschrieben werden. Dieser ergibt sich allein aus der Tatsache, dass nach Abzug der CAR weder vor, noch nach dem Stimulus irgendein Signal vorhanden ist. Daher kann der reale Gewinn im SNR hier mit etwa $6 \mathrm{~dB}$ geschätzt werden, was im Vergleich mit simulierten Daten ein akzeptabler Wert ist. Vergleicht man in Abb. 6 die Kanalverzögenungen untereinander, so kann festgestellt werden, dass einige Nachbarkanäle unwahrscheinliche Laufzeitdifferenzen aufweisen, z.B. Kanäle 9 und 10 bzw. 15 und 16 zeigen eine Differenz von mehr als $100 \mathrm{~ms}$. Dies kann durch die zu schwache Amplitude der VEP an den jeweiligen Sensoren verursacht werden. Daraus folgt, dass neben einer Adaptation im Zeitbereich auch eine Filterung im räumlichen Bereich nötig wäre. Mit einem (adaptiven) räumlichen Filter könnte der Einfluss der Kanäle mit niedrigem SNR reduziert werden.

Der vorgeschlagene Algorithmus funktioniert zuverlässig bei einphasigen transienten Signalen entsprechend der Simulation (Abb. 1 und Abb. 2). In diesem Fall weist die Abhängigkeit des SNR von der Kanalverzögerung nur ein Maximum auf, so dass die gradientbasierte iterative Suche (Gl. 10) zwangsläufig zur optimalen Lösung führt. In der Realität muss man aber davon ausgehen, dass die VEP ob unipolare oder bipolare bzw. nach CAR-Abzug mehrphasige Signale sind (d.h. mit mehreren Wellen). In diesem Fall ist das Optimum nicht unimodal, sondern je nach Anzahl der signifikanten Phasen des VEP treten mehrere lokale Maxima auf. Der vorgeschlagene Algorithmus kann lokale und globale Extremwerte nicht unterscheiden, daher muss nach abgeschlossener Adaptation nicht unbedingt das maximale SNR erreicht werden.

\section{Literaturverzeichnis}

[1] Paul L. Nunez, "Toward a quantitative description of large-scale neocortical dynamic function and EEG", Behavioral and Brain Sciences 23, 371-437, 2000

[2] Paul L.Nunez, Brett M. Wingeier, Richard B. Silberstein, "Spatial-Temporal Structures of Human Alpha Rhythms: Theory, Microcurrent Sources, Multiscale Measurements, and Global Binding of Local Networks", Human Brain Mapping 13, 125-164, 2001

[3] Husar, P., Berkes, S., Henning, G., Schellhorn, K., Schlegelmilch, F., Garner, D., "Beaming Signal Sources in Measurement of Focal Visual Evoked Cortical Potentials", 23rd Anual Itntl. Conf. of the IEEE EMBS 2001, Istanbul, Turkey, conf. proc. paper nr. 1098, 1-4, 2001 